\title{
Relaying for IEEE 802.11p at Road Intersection Using a Vehicular Non-Stationary Channel Model
}

\author{
Zhinan Xu*, Laura Bernadó*, Mingming Gan*, Markus Hofer*, Taimoor Abbas ${ }^{\dagger}$,

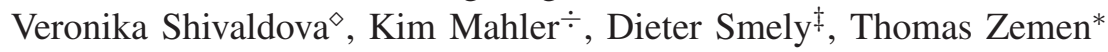 \\ ${ }^{*}$ FTW (Telecommunications Research Center Vienna), Vienna, Austria \\ ${ }^{\dagger}$ Department of Electrical and Information Technology, Lund University, Lund, Sweden \\ ${ }^{\diamond}$ Institute of Telecommunications, Vienna University of Technology, Vienna, Austria \\ $\div$ Fraunhofer Institute for Telecommunications, Heinrich Hertz Institute, Berlin, Germany \\ ${ }^{\ddagger}$ Kapsch TrafficCom AG, Vienna, Austria
}

\begin{abstract}
Traffic safety at road intersections can be improved by establishing reliable communications between vehicles. For vehicle-to-vehicle communications, this requires information exchange in non line-of-sight (NLOS) conditions due to the obstruction by buildings. In order to overcome the low receive signalto-noise ratio (SNR) due to NLOS, we consider to place a relay at road intersections to enhance the reliability of communication links. In this paper, we implement a vehicular non-stationary geometry based stochastic channel model for road intersections, which is an extension of an existing highway channel model. The model is verified by comparison with vehicular channel measurements. Using the proposed channel model, we present link level simulation results for IEEE 802.11p relaying at varying transmitter/receiver locations using different channel estimation techniques. The results show that a relay at the intersection is able to greatly extend the reliable communications region. Besides, in the high SNR regime with moderate or high mobility transmitter and receiver, the block type least square channel estimator is the bottleneck that limits the relaying performance. An advanced iterative channel estimator is also simulated, which exhibits robustness against increased vehicle velocities.
\end{abstract}

\section{INTRODUCTION}

In recent years, intelligent transportation systems (ITS) have received a lot of attention, since they enable safety-related applications, e.g. cross-traffic assistance and traffic condition warnings. The idea is that all vehicles exchange information, e.g., position and speed, with other vehicles periodically via cooperative awareness messages (CAM). Among all safety critical applications, the cross-traffic assistance in urban road intersections is one of the most challenging use cases due to the obstruction of the line-of-sight (LOS) by surrounding buildings. In case the LOS is blocked, specular and diffuse scatterings may enable non-line-of-sight (NLOS) reception, which in turn depends greatly on the availability of scattering objects at the intersection [1]. However, a high path-loss is expected due to the relatively high operating frequency [2]. In order to overcome the low signal-to-noise ratio (SNR) due to NLOS, we propose to deploy a relay at the intersection to enhance the reliability of communications. We confine this paper to the decode-and-forward (DF) protocol. From a practical implementation point of view, we consider only halfduplex relays, meaning that the relay is not able to transmit and receive simultaneously.
There exist a number of studies on traffic safety at road intersections [2]-[6]. A NLOS path-loss model for urban intersections is proposed in [2], and validated in [4]. The measured performance of $802.11 \mathrm{p}$ at intersections is studied in [5] and [6]. However, the measured results are only available at particular positions and speeds. In this paper, we aim at building up a controlled environment, such that the influences due to different factors, e.g. speed, position and channel estimation techniques, can be decoupled.

The contribution of this paper is as follows: i) We implement a geometry based stochastic model (GSCM) for road intersections, which is an extension of an existing highway channel model [7]. We parameterize and verify the model by comparing it with a measurement based path-loss model [2] and real-world vehicle-to-vehicle (V2V) channel measurements [8]. ii) We evaluate the communication performance in terms of frame error rate (FER) at various transmitter $(\mathrm{Tx}) /$ receiver $(\mathrm{Rx})$ locations and velocities with three different types of channel estimators. The influence of each factor is analyzed. iii) We present the performance evaluation with the aid of a DF relay. We show that a relay at the road intersection can strongly improve the SNR, whereas the high $\mathrm{Tx} / \mathrm{Rx}$ mobility can still lead to error propagation.

\section{SYSTEM OVERVIEW}

In this section we give a brief overview of the $802.11 \mathrm{p}$ standard, and describe the used relaying protocol, channel estimation techniques, and the non-stationary vehicular channel model.

\section{A. System Description}

We implemented a standard compliant IEEE 802.11 p physical layer OFDM transmission chain [9]. The transmission is frame based with frame length $M$ using $N$ subcarriers. The sampling rate is $R_{\mathrm{c}}$. A minimum mean squared error (MMSE) filter is used as the equalization method. Furthermore, due to the large carrier spacing $\Delta f=R_{\mathrm{c}} / N$ defined for $802.11 \mathrm{p}$, the inter-carrier interference is small enough to be neglected for the processing at the Rx side [10]. Accordingly, the received signal at subcarrier $k$ and time instant $n$ can be written as

$$
y[n, k]=h[n, k] x[n, k]+z[n, k],
$$




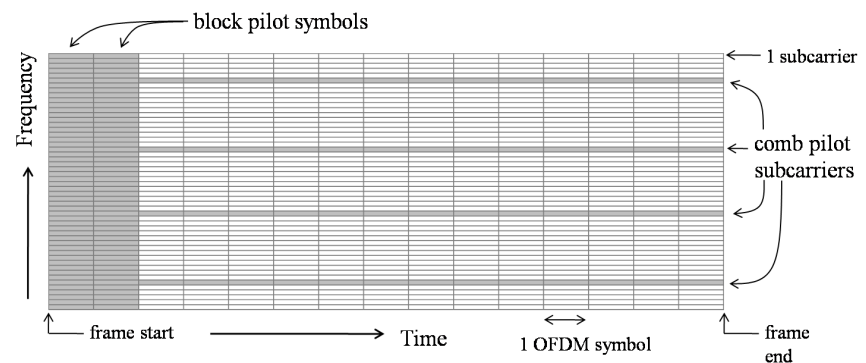

Fig. 1. Frame structure of IEEE 802.11p.

where $x[n, k]$ is the transmitted symbol, and $h[n, k]$ denotes the channel at time index $n$ and subcarrier $k$. Additive complex symmetric Gaussian noise at the receiver is denoted by $z[n, k] \sim \mathcal{C N}\left(0, \sigma^{2}\right)$.

\section{B. Channel Estimation Techniques}

The frame structure of IEEE 802.11p is shown in Fig. 1. All 52 subcarriers of the first two OFDM symbols are dedicated to pilots (block pilots). Afterwards, only 4 subcarriers contain pilots throughout the whole frame duration. They are known as comb pilot subcarriers, with subcarrier indices $\mathcal{I}_{\mathrm{c}}=\{6,20,33,47\}$. In the simulation of this paper, we consider the following three channel estimators.

- Block-type least square (BLS) channel estimator [11]: An estimate of the channel is calculated from the block pilots. This estimation technique is currently used for most commercial off-the-shelf receivers. Let us define the vectors containing the block pilot symbols as

$$
\begin{aligned}
& \mathbf{x}_{\mathrm{b} 1}=[x[0,0], \ldots, x[0, N-1]]^{\mathrm{T}}, \\
& \mathbf{x}_{\mathrm{b} 2}=[x[1,1], \ldots, x[1, N-1]]^{\mathrm{T}},
\end{aligned}
$$

and the concatenated vector of the received signal at block pilot positions as

$$
\begin{aligned}
\mathbf{y}_{\mathrm{b}}= & {[y[0,0], \ldots, y[0, N-1],} \\
& y[1,0], \ldots, y[1, N-1]]^{\mathrm{T}} .
\end{aligned}
$$

The channel estimates are obtained as

$$
\hat{\mathbf{h}}=\left(\mathbf{X}_{\mathrm{b}}^{\mathrm{H}} \mathbf{X}_{\mathrm{b}}\right)^{-1} \mathbf{X}_{\mathrm{b}}^{\mathrm{H}} \mathbf{y}_{\mathrm{b}},
$$

where $\mathbf{X}_{\mathrm{b}}=\left[\operatorname{diag}\left(\mathbf{x}_{\mathrm{b} 1}\right), \operatorname{diag}\left(\mathbf{x}_{\mathrm{b} 2}\right)\right]^{\mathrm{T}}$. The estimated channel coefficients are then used for the whole frame assuming a block fading channel.

- Block-comb-type MMSE (BC-MMSE) channel estimator [9]: An initial estimate of the channel is calculated from the comb pilots using the least square estimation. Subsequently, a linear MMSE filtering is performed in the time domain. The channel coefficients at comb pilot positions are defined as

$$
\mathbf{h}_{\mathrm{c} i}=\left[x\left[0, \mathcal{I}_{\mathrm{c}}(i)\right], \ldots, x\left[M-1, \mathcal{I}_{\mathrm{c}}(i)\right]\right]^{\mathrm{T}} .
$$

The MMSE estimate of the channel matrix is obtained as

$$
\hat{\mathbf{H}}=\hat{\mathbf{R}} \mathbf{X}_{\mathrm{b}}^{\mathrm{H}}\left(\mathbf{X}_{\mathrm{b}} \hat{\mathbf{R}} \mathbf{X}_{\mathrm{b}}^{\mathrm{H}}+\sigma^{2} \mathbf{I}\right)^{-1} \mathbf{Y},
$$

where $\mathbf{Y}$ is the matrix containing the received symbols at pilot positions. The channel time correlation matrix is estimated as

$$
\hat{\mathbf{R}}=\frac{1}{4} \sum_{i=1}^{4} \hat{\mathbf{h}}_{\mathrm{c} i} \hat{\mathbf{h}}_{\mathrm{c} i}^{\mathrm{H}}
$$

where $\hat{\mathbf{h}}_{\mathrm{c} i}$ is the least square estimate of $\mathbf{h}_{\mathrm{c} i}$.

- Iterative channel estimator based on discrete prolate spheroidal sequences (DPSs) in the time and frequency domain [10] [12] (referred to as iterative DPS channel estimator in the rest of the paper): The channel estimation is performed using a reduced-rank MMSE equalizer. The output of the equalizer is used as input to a BCJR decoder [13] after de-mapping and de-interleaving. The soft-output information from the BCJR decoder is used iteratively to obtain a better channel estimate.

\section{Relaying Techniques}

In the relaying context, $\mathrm{Tx}$ and $\mathrm{Rx}$ are referred to as source and destination, respectively. We consider a system with a moving source, a static relay and a moving destination. The relay is static at the intersection in which the exchange of information can be safety critical. The moving source and destination travel at velocities $v_{\mathrm{s}}$ and $v_{\mathrm{d}}$, respectively. In this paper, we consider a time division duplex (TDD) relaying strategy, where the data transmission consists of two phases with equal time duration. During the first phase, the source broadcasts the modulated signal towards the relay and the destination. The received signals at the relay and the destination are

$$
\begin{aligned}
& y_{\mathrm{SR}}[n, k]=h_{\mathrm{SR}}[n, k] x[n, k]+z_{\mathrm{SR}}[n, k] \\
& y_{\mathrm{SD}}[n, k]=h_{\mathrm{SD}}[n, k] x[n, k]+z_{\mathrm{SD}}[n, k]
\end{aligned}
$$

where $h_{\mathrm{SR}}[n, k]$ and $h_{\mathrm{SD}}[n, k]$ denote the channels from source to relay (SR) and source to destination (SD) at time index $n$ and subcarrier $k$, respectively. Additive complex symmetric Gaussian noise at the relay and destination is denoted as $z_{\mathrm{SR}}[n, k] \sim \mathcal{C N}\left(0, \sigma^{2}\right)$ and $z_{\mathrm{SD}}[n, k] \sim \mathcal{C N}\left(0, \sigma^{2}\right)$.

Then, the detected symbols $\hat{x}[n, k]$ at the relay are reencoded and transmitted to the destination in the second phase. The received signal at the destination is

$$
y_{\mathrm{RD}}[n, k]=h_{\mathrm{RD}}[n, k] \hat{x}[n, k]+z_{\mathrm{RD}}[n, k],
$$

where $h_{\mathrm{RD}}[n, k]$ denotes the channel from relay to destination (RD) at time index $n$ and subcarrier $k$. Additive complex symmetric Gaussian noise at the destination is denoted by $z_{\mathrm{RD}}[n, k] \sim \mathcal{C N}\left(0, \sigma^{2}\right)$.

At the destination, the signal received from the source during the first phase, and the signal received from the relay during the second phase, are combined using maximum ratio combining (MRC) [14]. Defining $\mathbf{y}=\left[y_{\mathrm{RD}}, y_{\mathrm{SD}}\right]^{\mathrm{T}}$ and $\mathbf{z}=\left[z_{\mathrm{RD}}, z_{\mathrm{SD}}\right]^{\mathrm{T}}$, the combined signal at the destination is

$$
r[n, k]=\mathbf{u}^{\mathrm{H}}[n, k] \mathbf{y}[n, k] .
$$


The combining weights are calculated as

$$
\mathbf{u}[n, k]=\frac{\left[\hat{h}_{\mathrm{RD}}[n, k], \hat{h}_{\mathrm{SD}}[n, k]\right]^{\mathrm{T}}}{\left\|\left[\hat{h}_{\mathrm{RD}}[n, k], \hat{h}_{\mathrm{SD}}[n, k]\right]\right\|},
$$

where $\hat{h}_{\mathrm{SD}}$ and $\hat{h}_{\mathrm{RD}}$ are the estimates of $h_{\mathrm{SD}}$ and $h_{\mathrm{RD}}$.

\section{NON-STATIONARY CHANNEL MODEL}

In order to obtain realistic simulation results, we need a channel model that resembles the true propagation conditions. The commonly used tap delay line channel model [15] for 802.11p does not accurately represent the most important features of vehicular channels [7]. As shown by vehicular radio channel measurements at $5 \mathrm{GHz}$, the channel impulse response of vehicular channels is mainly composed of: LOS, deterministic scattering, and diffuse scattering. A very important characteristic of vehicular channels is that different delays present correlated fading due to reflections coming from the same object along time. Furthermore, the statistical properties of the channel change over time and therefore the channel is non-stationary. For this reason, we use a nonstationary GSCM for road intersections and parameterize it from vehicular measurements [2], [8].

As shown in Fig. 2, we generate a typical road crossing scenario, which consists of a moving Tx, a moving $\mathrm{Rx}$, some mobile and static discrete scatterers (MD and SD) and diffuse scatterers (D) at the two sides of the road. The MD scatterers represent other cars moving on the road, while the SD scatterers represent traffic signs and parked cars. The diffuse scattering wall models foliage and other objects along the road.

The function that maps the subcarrier index $k \in$ $\{0, \ldots, N-1\}$ into the discrete frequency index is defined as $\varphi(k)=((k+N / 2 \bmod N)-N / 2)$. The time-variant channel frequency response is generated as

$$
\begin{aligned}
& h[n, k]= \\
& \alpha^{(\mathrm{LOS})}\left(n T_{\mathrm{c}}\right) \gamma^{(\mathrm{LOS})}\left(n T_{\mathrm{c}}\right) \exp \left[-j 2 \pi \Delta f \varphi(k) \tau^{(\mathrm{LOS})}\left(n T_{\mathrm{c}}\right)\right]+ \\
& \sum_{p=1}^{N_{\mathrm{SD}}} \alpha_{p}^{(\mathrm{SD})}\left(n T_{\mathrm{c}}\right) \gamma_{p}^{(\mathrm{SD})}\left(n T_{\mathrm{c}}\right) \exp \left[-j 2 \pi \Delta f \varphi(k) \tau_{p}^{(\mathrm{SD})}\left(n T_{\mathrm{c}}\right)\right]+ \\
& \sum_{p=1}^{N_{\mathrm{MD}}} \alpha_{p}^{(\mathrm{MD})}\left(n T_{\mathrm{c}}\right) \gamma_{p}^{(\mathrm{MD})}\left(n T_{\mathrm{c}}\right) \exp \left[-j 2 \pi \Delta f \varphi(k) \tau_{p}^{(\mathrm{MD})}\left(n T_{\mathrm{c}}\right)\right]+ \\
& \sum_{p=1}^{N_{\mathrm{D}}} \alpha_{p}^{(\mathrm{D})}\left(n T_{\mathrm{c}}\right) \gamma_{p}^{(\mathrm{D})}\left(n T_{\mathrm{c}}\right) \exp \left[-j 2 \pi \Delta f \varphi(k) \tau_{p}^{(\mathrm{D})}\left(n T_{\mathrm{c}}\right)\right]
\end{aligned}
$$

where $\gamma_{p}^{(\cdot)}$ denote the complex-valued attenuation coefficients of different paths, which takes into account the effects of path loss, antenna radiation patterns and large-scale fading, $\tau_{p}^{(\cdot)}$ denote the delays of the paths, and $N_{(\cdot)}$ are the numbers of SD, MD and D scatterers, respectively. The existence of different paths is represented by $\alpha_{p}^{(\cdot)}$, which equals to 0 if the path is blocked by the buildings, and to 1 otherwise.

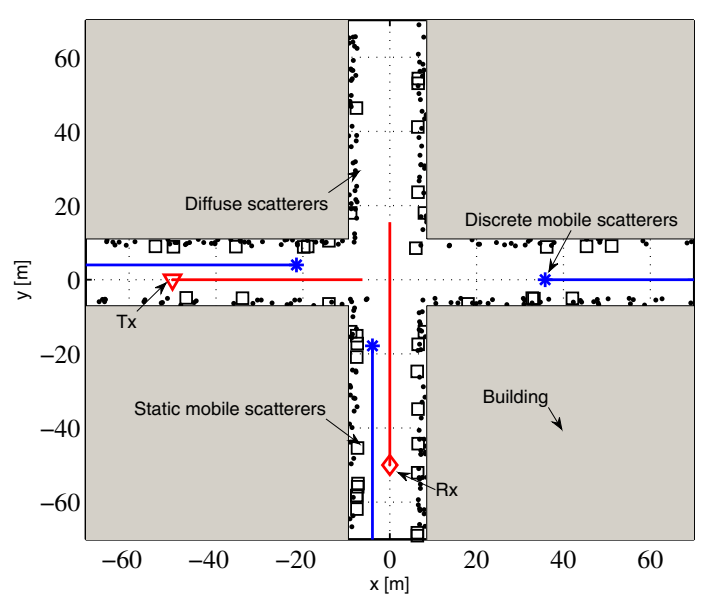

Fig. 2. Scatterers distribution of the proposed channel model.

TABLE I

MODIFICATIONS TO THE PARAMETERS RELATIVE TO [7, TABLE 1]

\begin{tabular}{lcccc}
\hline Parameter $^{1}$ & LOS & MD & SD & D \\
\hline$G_{0}[\mathrm{~dB}]$ & -5 & $-89+24 n$ & $-89+24 n$ & 50 \\
$n$ & 1.8 & $\mathcal{U}[3.5,5.5]$ & $\mathcal{U}[3.5,5.5]$ & 5.4 \\
$\chi\left[\mathrm{m}^{-1}\right]$ & - & 0.01 & 0.3 & 1 \\
$W_{\mathrm{DI}}[\mathrm{m}]$ & - & - & - & 2 \\
\hline
\end{tabular}

1 The detailed definition of the parameters can be found in [7].

\section{A. Validation of the Proposed Model}

In order to parametrize and validate the model, we need real-world radio channel measurements. For that, we use the measurements collected in a $\mathrm{V} 2 \mathrm{~V}$ measurement campaign carried out in an urban crossing environment in Lund city, Sweden [8], shown in Fig. 4. The measurements in [8] are collected using a center frequency of $5.6 \mathrm{GHz}$ and a bandwidth of $240 \mathrm{MHz}$. Therefore, we first need to tune our GSCM to these RF parameters and then proceed with the parametrization and validation of the scattering coefficients. Once the GSCM parameters are set and validated, we re-tune the center frequency and the bandwidth to the ones defined in the $802.11 \mathrm{p}$ standard for link-level simulations.

As shown in Fig. 4, the investigated intersection consists of two streets, each having a width of $17 \mathrm{~m}$. The distance to the wall on the right side is $6 \mathrm{~m}$. The Tx and $\mathrm{Rx}$ are traveling towards the intersection from $50 \mathrm{~m}$ away with a speed of $20 \mathrm{~km} / \mathrm{h}$ and $30 \mathrm{~km} / \mathrm{h}$, respectively. The time duration of each run is $8 \mathrm{~s}$. In Table I we list the modifications of the parameters relative to $[7$, Table 1$]$.

We use two metrics to validate this parametrization: the path-loss and the root-mean-square (RMS) delay spread. First, we compare in Fig. 3 the normalized path-gain of our simulated channel to the measurement based path-loss model for road intersections derived in [2] using the same data. For simulated channels, we show 20 independent simulation runs. The small scale fading has been removed by averaging the data 


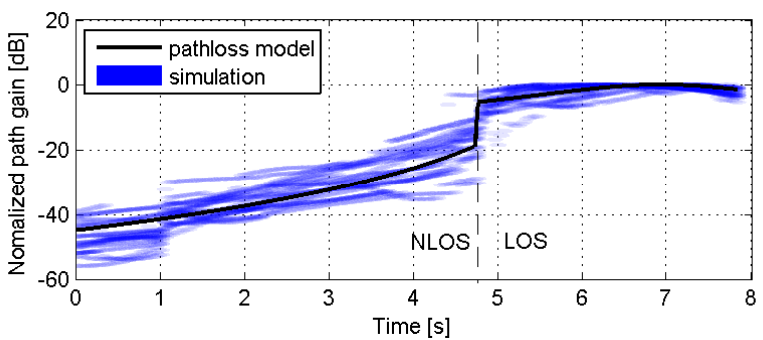

Fig. 3. Comparison of the normalized path-loss of the measurement based pathloss model and the simulated channel.

over the stationary region using the local scattering function estimator [16], whereas the large-scale fading is still preserved, which results in the fluctuations around the curve of the pathloss model. By comparing to the pathloss model, we observe that the distance-dependent decay of the path gain in the NLOS region is greater for intersection scenarios than for highway scenarios [7]. Thus, we modify the distribution of the pathloss exponents to $\mathcal{U}[2,5.5]$ for discrete scatterers in order to get a better fit.

Subsequently, we validate the model using the RMS delay spread. The delay spread has a strong impact on the Rx performance [9]. However, in most channel models, it is assumed to be constant. Non-stationary vehicular channels result in a time-varying delay spread [16]. Thus, we calculate the root mean square (RMS) delay spread, and compare it with the result obtained from channel measurements [8] performed in the city of Lund, Sweden. The selected intersection with the trajectories of the Tx and the Rx is shown in Fig. 4. In Fig. 5, it can be observed that the delay spread of the simulated channel fits well with the measurements in the NLOS region and is slightly smaller than the measurements in the LOS region. This is due to the richness of multipath components present near the intersection. In order to maintain a low computational complexity in the GSCM, we only include first-order reflection paths. Nevertheless, the results obtained from simulation and measurements are close enough to consider the model valid.

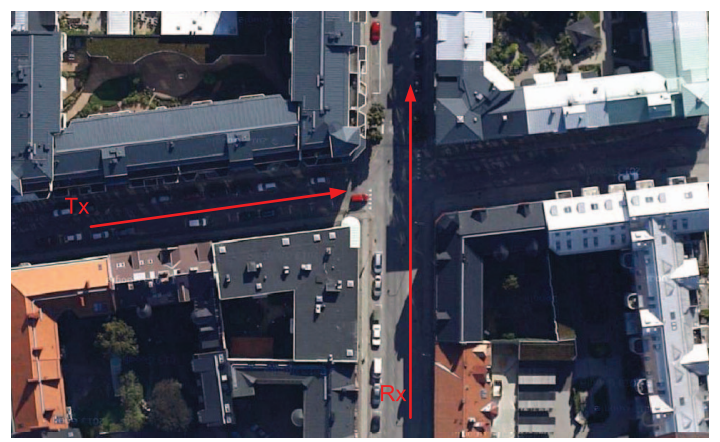

Fig. 4. Top view of the investigated intersection (N55 $\left.42^{\prime} 38^{\prime \prime}, \mathrm{E} 13^{\circ} 11^{\prime} 14^{\prime \prime}\right)$ in the city of Lund with the trajectories of $\mathrm{Tx}$ and $\mathrm{Rx}$ respectively.

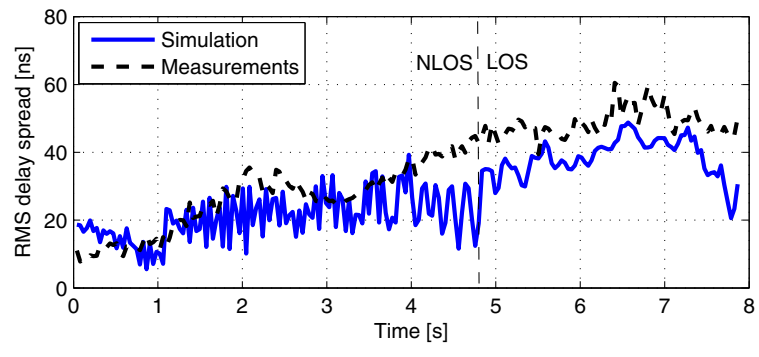

Fig. 5. Comparison of the RMS delay spread of the measured channel and the simulated channel.

TABLE II

PARAMETERS FOR TRANSMISSION

\begin{tabular}{ll}
\hline Parameter & Value \\
\hline Transmit power $[\mathrm{dBm}]$ & 20 \\
Antenna gain [dB] & 0 \\
System loss [dB] & 5 \\
Data rate [Mbps] & $6(\mathrm{QPSK})$ \\
Frame length [Bytes], $M$ & 200 \\
Bandwidth [MHz], $T_{\mathrm{c}}$ & 10 \\
Noise power [dBm], $\sigma^{2}$ & -104 \\
\hline
\end{tabular}

\section{Simulation Results}

Using the channel model parameterized in Sec. III, we re-tune the center frequency and bandwidth according to the $802.11 \mathrm{p}$ system parameters. The simulation is performed at varying $\mathrm{Tx} / \mathrm{Rx}$ positions and velocities. For each different combination, 100 frames are simulated. We choose the $802.11 \mathrm{p}$ coding and modulation scheme achieving $6 \mathrm{Mbps}$, which is one of the most robust transmission schemes. It employs quadrature phase shift keying (QPSK) modulation and a convolutional code with constraint length 7 at a coding rate of $1 / 2$. Table II lists the detailed parameters of the simulation. The communication performance in terms of FER is presented in this section.

\section{A. Performance without Relaying}

In this section, we present the results of direct transmission without relaying. Fig. 6 shows the FER results using the BLS channel estimator at varying $\mathrm{Tx}$ and $\mathrm{Rx}$ position with different velocities. For the investigated scenario, the LOS is only available at the lower left corner when both vehicles are $10 \mathrm{~m}$ away from the intersection. As expected, the FER is strongly distance dependent. When both cars are far away from the intersection $(>50 \mathrm{~m}$ ), almost no frame can be successfully delivered due to the low SNR (power-limited scenario). It is noteworthy that if one vehicle is close to the intersection, e.g., $<10 \mathrm{~m}$, a high enough SNR can be guaranteed regardless of the position of the other vehicle. Thus, the FER is almost independent of the position of the other vehicle. In this region, the FER increases with the velocities of the vehicles (velocitylimited scenario). This is due to the fact that the channel estimates are acquired only from the block pilots and used for the whole frame. However, the channel changes after the transmission of the pilots which leads to an increased FER 

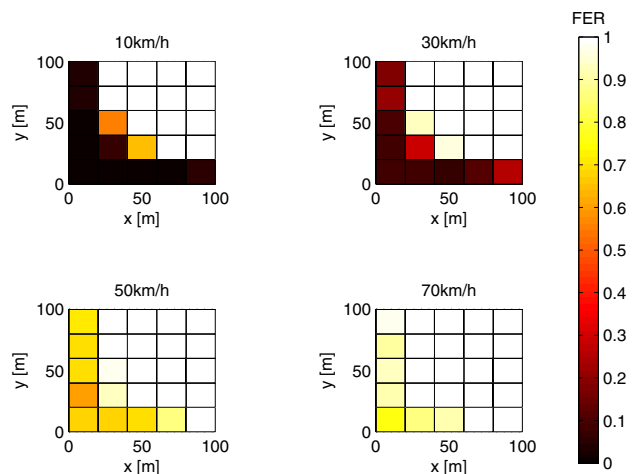

Fig. 6. FER results without relaying using the BLS estimator, at different Tx and $\mathrm{Rx}$ positions $(\{10,30,50,70,90\} \mathrm{m}$ to the center $)$.
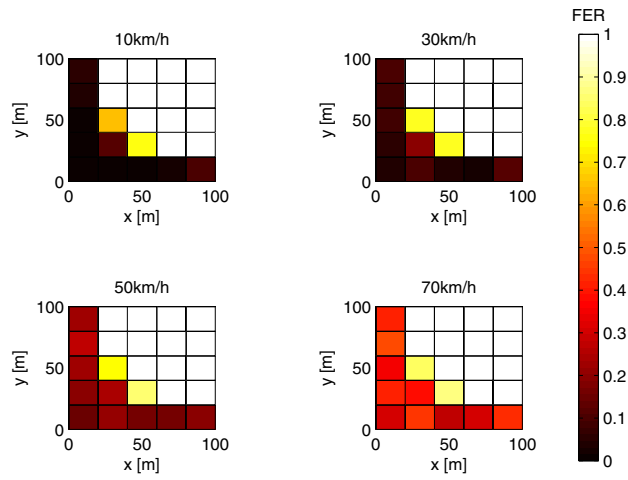

Fig. 7. FER results without relaying using the BC-MMSE estimator, at different $\mathrm{Tx}$ and $\mathrm{Rx}$ positions $(\{10,30,50,70,90\} \mathrm{m}$ to the center).

due to the use of outdated channel estimates.

The increase of FER due to high mobility is relieved using BC-MMSE channel estimator, as shown in Fig. 7. The estimated time-correlation matrix provides a good estimate of the time evolution for channels with low delay spread. This improves the performance of a block pilot based channel estimator.

To further combat the degradation caused by high mobility, a more advanced signal processing algorithm at the $\mathrm{Rx}$ has to be used. In Fig. 8, we show the FER results using the iterative DPS channel estimator with 5 iterations. It can be seen that the DPS channel estimator is robust to higher velocities. Compared to Fig. 6, the FER is improved, which gives an extended possible communication region. As indicated in [17], a brake warning should be given at approximately $3 \mathrm{~s}$ to a potential collision. If the vehicles travel at $70 \mathrm{~km} / \mathrm{h}$, the communication between vehicles has to be established at a distance of around $60 \mathrm{~m}$, which is still very challenging for the current setup.

\section{B. Performance with a Relay at the Intersection Center}

In order to further enhance the communication performance, we place a fixed DF relay at the center of the intersection (at
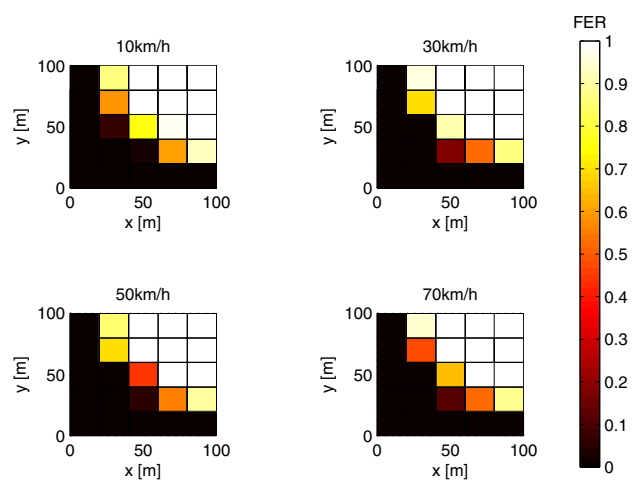

Fig. 8. FER results without relaying using the iterative DPS estimator, at different $\mathrm{Tx}$ and $\mathrm{Rx}$ positions $(\{10,30,50,70,90\} \mathrm{m}$ to the center).

the origin in Fig. 2) and assume LOS between the relay and the two vehicles. The transmission parameters of the relay are given in Table II. MRC is used at the $\mathrm{Rx}$ to combine the signals received from the Tx and relay. Fig. 9 shows the FER with relaying and BLS channel estimator. It can be seen that the entire region becomes almost distance-independent because the relay guarantees a high SNR for the SR link and the RD link. In this region, almost all frames are successfully received at a speed of $10 \mathrm{~km} / \mathrm{h}$, while for the vehicle velocity of $70 \mathrm{~km} / \mathrm{h}$ the error rate increases to around $70 \%$. Therefore, in the high SNR regime with high mobility of the Tx and the $\mathrm{Rx}$, the use of the BLS estimator limits the performance.

In Fig. 10, the BC-MMSE estimator provides better performance by accounting for the time variability of the channel. The improvement is considerable compared to BLS estimation, especially if all sub-channels fade similarly (e.g., a channel with strong LOS component and low delay spread). We can observe slightly higher FER when a vehicle is near the intersection. This is because more multipath components are enabled near the intersection, which degrades the performance due to the increase of delay- and Doppler-spreads. If the iterative DPS estimator is used together with a relay in the intersection center, almost error-free transmission can be achieved even at 70km/h, as shown in Fig. 11 .

\section{CONCLUSiON}

We implemented a vehicular non-stationary channel model for road intersections. The proposed model fits well with the $\mathrm{V} 2 \mathrm{~V}$ channel measurements. Using the proposed channel model, we showed link level simulation results for IEEE 802.11 p relaying at road intersections with varying $\mathrm{Tx} / \mathrm{Rx}$ locations using different channel estimation techniques. We found that a relay at the intersection greatly extends the reliable communication region. Furthermore, the velocity of the Tx and the Rx has a strong impact on the achieved FER if the block-type least square channel estimator is used. For velocities higher than $50 \mathrm{~km} / \mathrm{h}$, a block-comb-type estimator or an advanced iterative DPS channel estimator [12] ensures a 

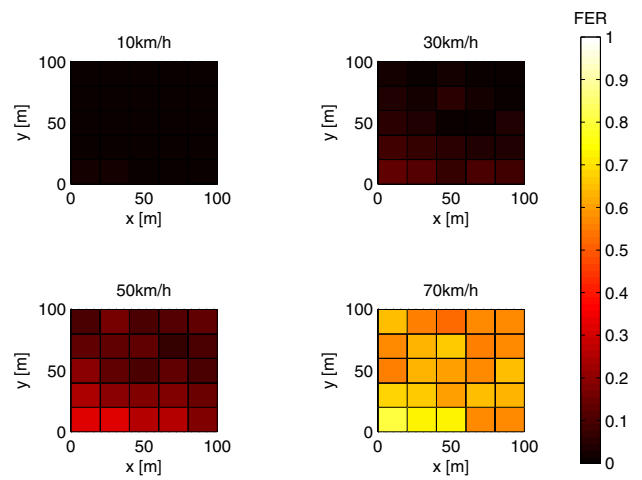

Fig. 9. FER results with relaying using the BLS estimator, at different Tx and $\mathrm{Rx}$ positions $(\{10,30,50,70,90\} \mathrm{m}$ to the center).
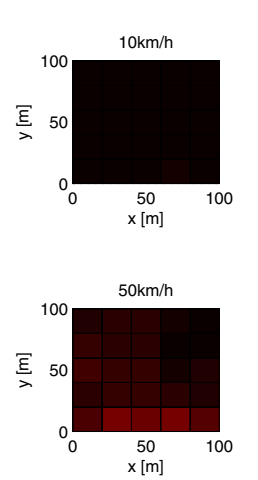

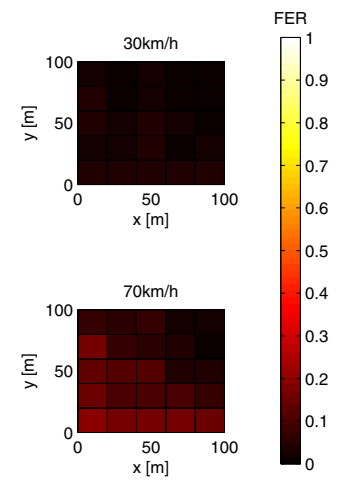

Fig. 10. FER results with relaying using the BC-MMSE estimator, at different $\mathrm{Tx}$ and $\mathrm{Rx}$ positions $(\{10,30,50,70,90\} \mathrm{m}$ to the center).

low FER and a large distance range in safety critical crossing scenarios.

\section{ACKNOWLEDGMENT}

This work was performed in the FTW project ITS Evolution, a scientific cooperation co-funded by ASFINAG, Kapsch TrafficCom AG and Vienna University of Technology. The Austrian Competence Center FTW Forschungszentrum Telekommunikation Wien $\mathrm{GmbH}$ is funded within the program COMET - Competence Centers for Excellent Technologies by BMVIT, BMWFJ, and the City of Vienna. The COMET program is managed by the FFG.

\section{REFERENCES}

[1] J. Karedal, F. Tufvesson, T. Abbas, O. Klemp, A. Paier, L. Bernadó, and A. F. Molisch, "Radio channel measurements at street intersections for vehicle-to-vehicle safety applications," in IEEE 71st Veh. Technol. Conf. IEEE, 2010, pp. 1-5.

[2] T. Mangel, O. Klemp, and H. Hartenstein, "A validated $5.9 \mathrm{GHz}$ nonline-of-sight path-loss and fading model for inter-vehicle communication," in 11th Int. Conf. ITS Telecommun., Aug. 2011, pp. 75-80.

[3] K. Mahler, P. Paschalidis, M. Wisotzki, A. Kortke, and W. Keusgen, "Evaluation of vehicular communication performance at street intersections," Submitt. to VTC Fall - IEEE Veh. Technol. Conf., 2014.
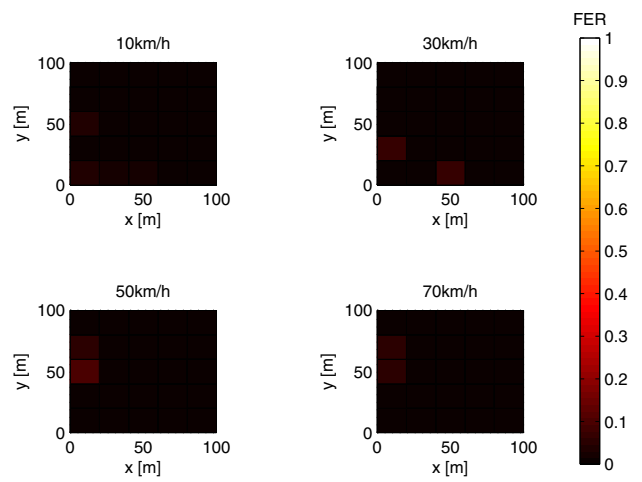

Fig. 11. FER results with relaying using the iterative DPS estimator, at different $\mathrm{Tx}$ and $\mathrm{Rx}$ positions $(\{10,30,50,70,90\} \mathrm{m}$ to the center).

[4] T. Abbas, A. Thiel, T. Zemen, C. F. Mecklenbrauker, and F. Tufvesson, "Validation of a non-line-of-sight path-loss model for V2V communications at street intersections," in 13th Int. Conf. ITS Telecommun., Nov. 2013, pp. 198-203.

[5] M. Schack, J. Nuckelt, R. Geise, L. Thiele, and T. Kurner, "Comparison of path loss measurements and predictions at urban crossroads for $\mathrm{C} 2 \mathrm{C}$ communications," in Antennas Propag. (EUCAP), Proc. 5th Eur. Conf., 2011, pp. 2896-2900.

[6] H. Schumacher, H. Tchouankem, J. Nuckelt, T. Kürner, T. Zinchenko, A. Leschke, and L. Wolf, "Vehicle-to-vehicle IEEE 802.11p performance measurements at urban intersections," in IEEE Int. Conf. Commun., Jun. 2012, pp. 7131-7135.

[7] J. Karedal, F. Tufvesson, N. Czink, A. Paier, C. Dumard, T. Zemen, C. F. Mecklenbräuker, and A. Molisch, "A geometry-based stochastic MIMO model for vehicle-to-vehicle communications," IEEE Trans. Wirel. Commun., vol. 8, no. 7, pp. 3646-3657, Jul. 2009.

[8] A. Paier, L. Bernadó, J. Karedal, O. Klemp, and A. Kwoczek, "Overview of vehicle-to-vehicle radio channel measurements for collision avoidance applications," in 2010 IEEE 71st Veh. Technol. Conf., 2010, pp. 1-5.

[9] L. Bernadó, N. Czink, T. Zemen, and P. Belanovic, "Physical layer simulation results for IEEE $802.11 \mathrm{p}$ using vehicular non-stationary channel model," in 2010 IEEE Int. Conf. Commun. Work., May 2010, pp. 1-5.

[10] T. Zemen, L. Bernadó, N. Czink, and A. F. Molisch, "Iterative timevariant channel estimation for $802.11 \mathrm{p}$ using generalized discrete prolate spheroidal sequences," IEEE Trans. Veh. Technol., vol. 61, no. 3, pp. 1222-1233, Mar. 2012.

[11] J. van de Beek, O. Edfors, M. Sandell, S. Wilson, and P. Borjesson, "On channel estimation in OFDM systems," in IEEE 45th Veh. Technol. Conf. Countdown to Wirel. Twenty-First Century, vol. 2, no. 1, 1995, pp. $815-819$.

[12] T. Zemen and A. F. Molisch, "Adaptive reduced-rank estimation of nonstationary time-variant channels using subspace selection," IEEE Trans. Veh. Technol., vol. 61, no. 9, pp. 4042-4056, Nov. 2012.

[13] L. Bahl, J. Cocke, F. Jelinek, and J. Raviv, "Optimal decoding of linear codes for minimizing symbol error rate," IEEE Trans. Inf. Theory, vol. 20, no. 2, pp. 284-287, Mar. 1974.

[14] D. Brennan, "Linear diversity combining techniques," Proc. IEEE, vol. 91, no. 2, pp. 331-356, Feb. 2003.

[15] G. Acosta-Marum and M. Ingram, "Six time- and frequency- selective empirical channel models for vehicular wireless LANs," IEEE Veh. Technol. Mag., vol. 2, no. 4, pp. 4-11, Dec. 2007.

[16] L. Bernadó, T. Zemen, F. Tufvesson, A. F. Molisch, and C. F. Mecklenbräuker, "Delay and Doppler spreads of nonstationary vehicular channels for safety-relevant scenarios," IEEE Trans. Veh. Technol., vol. 63, no. 1, pp. 82-93, Jan. 2014.

[17] C. Grover, I. Knight, and F. Okoro, "Automated emergency brake systems: Technical requirements, costs and benefits," Tech. Rep., 2013. 